\title{
When fast logic meets slow belief: Evidence for a parallel-processing model of belief bias
}

\author{
Dries Trippas ${ }^{1} \cdot$ Valerie A. Thompson ${ }^{2} \cdot$ Simon J. Handley ${ }^{3}$ \\ Published online: 27 December 2016 \\ (C) The Author(s) 2016. This article is published with open access at Springerlink.com
}

\begin{abstract}
Two experiments pitted the default-interventionist account of belief bias against a parallel-processing model. According to the former, belief bias occurs because a fast, belief-based evaluation of the conclusion pre-empts a working-memory demanding logical analysis. In contrast, according to the latter both belief-based and logic-based responding occur in parallel. Participants were given deductive reasoning problems of variable complexity and instructed to decide whether the conclusion was valid on half the trials or to decide whether the conclusion was believable on the other half. When belief and logic conflict, the default-interventionist view predicts that it should take less time to respond on the basis of belief than logic, and that the believability of a conclusion should interfere with judgments of validity, but not the reverse. The parallelprocessing view predicts that beliefs should interfere with logic judgments only if the processing required to evaluate the logical structure exceeds that required to evaluate the knowledge necessary to make a belief-based judgment, and vice versa otherwise. Consistent with this latter view, for the simplest reasoning problems (modus ponens), judgments of belief resulted in lower accuracy than judgments of validity, and believability
\end{abstract}

Electronic supplementary material The online version of this article (doi:10.3758/s13421-016-0680-1) contains supplementary material, which is available to authorized users.

Dries Trippas

trippas@mpib-berlin.mpg.de

1 Center for Adaptive Rationality, Max Planck Institute for Human Development, Berlin, Germany

2 Department of Psychology, University of Saskatchewan, Saskatoon, Saskatchewan, Canada

3 Faculty of Human Sciences, Macquarie University, Sydney, NSW, Australia interfered more with judgments of validity than the converse. For problems of moderate complexity (modus tollens and single-model syllogisms), the interference was symmetrical, in that validity interfered with belief judgments to the same degree that believability interfered with validity judgments. For the most complex (three-term multiple-model syllogisms), conclusion believability interfered more with judgments of validity than vice versa, in spite of the significant interference from conclusion validity on judgments of belief.

Keywords Deductive reasoning $\cdot$ Conflict detection $\cdot$ Dual process theory $\cdot$ Logic $\cdot$ Belief

The effects of beliefs on logical reasoning are pervasive and have been investigated for almost nine decades (Wilkins, 1929). The believability of conclusions influences how arguments are evaluated across a wide range of paradigms. Believable conclusions are deemed more acceptable than unbelievable ones regardless of logical validity (Evans, Barston, \& Pollard, 1983), regardless of the strength of the arguments (Stanovich \& West, 1997), and regardless of whether the task involves formal or informal reasoning (Thompson \& Evans, 2012). The goal of the current paper is to test predictions from two competing theoretical accounts of belief bias in deductive reasoning, both of which can be placed under the wider metatheoretical framework of dual process theories of reasoning.

According to dual process theory, two types of qualitatively different cognitive processes can be distinguished: Type 1 processes are autonomous and Type 2 processes require working memory (Evans \& Stanovich, 2013a). According to the default-interventionist instantiation of dual process theory, Type 1 processes cue default responses which may then be overridden by Type 2 processes. The default-interventionist account further assumes that beliefs are accessible to Type 1 
processing, but that accurate logical reasoning requires Type 2 processing (Evans \& Stanovich, 2013b). Consequently, belief effects arise because the (generally faster) Type 1 processes substitute an answer based on belief for one based on logical validity (Evans \& Curtis-Holmes, 2005).

Recently, however, theorists have acknowledged that this characterization is too simple for a number of reasons (see e.g., Kruglanski \& Gigerenzer, 2011). Equating Type 1 processes with bias and Type 2 processing with normative reasoning is a fallacy (Elqayam \& Evans, 2011; Evans \& Stanovich, 2013a; Thompson, Prowse Turner, \& Pennycook, 2011): Type 1 processes produce errors on some occasions and correct responses on others, as do Type 2 processes. This is empirically supported by evidence that judgments based on formal norms such as logic and probability (traditionally equated with Type 2 processing) may be made quickly and implicitly, suggesting that these judgments may, in some instances, arise from Type 1 processes. For example, recent evidence suggests that some types of simple logical arguments are processed autonomously (Bago \& De Neys, 2017; Morsanyi \& Handley, 2012; Trippas, Handley, Verde, \& Morsanyi, 2016; though see Klauer \& Singmann, 2013). Similarly, although belief judgments have been shown to be made rapidly and accurately, it is well established that these involve some form of inferential processing distinct from pure fact-retrieval from memory (Reder, 1982). Furthermore, the automaticity of belief judgments depends on various mediating factors such as mind-set and task demands (Wiswede, Koranyi, Mueller, Langner, \& Rothermund, 2013). A review of the available evidence suggests that occasionally belief judgments may require considerable time and effort (cf., Handley \& Trippas, 2015), suggesting that here too a pure classification in terms of Type 1 or Type 2 processing is overly simplistic. Taken together, these data support models in which logical and belief-based processing is initiated simultaneously (De Neys, 2012, 2014; Handley \& Trippas, 2015; Pennycook, Fugelsang, \& Koehler, 2015; Sloman, 2014).

Direct evidence for this position comes from a pair of studies by Handley, Newstead, and Trippas (2011) and Pennycook, Trippas, Handley, and Thompson (2014). Handley and colleagues instructed reasoners to evaluate conclusions to very simple logical arguments of the modus ponens form (i.e., if $p$, then q; $p$, therefore $q$ ). The novel element of the task was that they were asked to provide one of two judgments: on one half of the trials, participants had to evaluate the validity of the conclusion (as is traditionally done), but on the other half, they had to evaluate the believability of the conclusion (as has been investigated extensively in research on truth verification, cf. Reder, 1982). When the two sources of information conflicted, it was found that the validity of the syllogism interfered with the ability to make belief judgments, as evidenced by higher error rates and longer response times for conflict than non-conflict problems.
This pattern would not be expected if judgments of validity took longer or were more difficult than judgments of belief. Pennycook and colleagues replicated this finding using a completely different task, modelled on Tversky and Kahneman's (1973) base-rate task. Participants were provided with the base-rate probability of category membership (e.g., $5 \%$ of the people in this sample are engineers and $95 \%$ are lawyers) and a personality description of an individual (e.g., John is a great computer programmer and loves board games). Again, when the two sources of information conflicted, the base-rate information interfered with making belief-based judgments, which is inconsistent with the view that the latter form a fast, default response (Kahneman 2011).

Collectively, these data seem to support parallel processing (De Neys, 2012; Handley \& Trippas, 2015; Pennycook et al., 2015; Sloman, 2014) in that multiple relevant problem features (e.g., structure and belief content) may be processed simultaneously. In the case where both problem aspects can be assessed in a relatively simple way, they cause mutual interference. However, in cases where one or the other response requires more complex processing, an asymmetry should arise (Handley \& Trippas, 2015). According to the parallel-processing model, it is the complexity of the relevant problem features that determines response accuracy and speed. Logical judgments superseded belief judgments in the cases presented by Handley et al. (2011) due to the relative simplicity of the logical structure versus the somewhat more moderate complexity of the belief judgments. This directly implies that as the logical judgments become more complex, the interference should reverse - with conflict affecting logic judgments more than belief judgments.

In support of this hypothesis, some studies show that the extent of belief bias observed varies as a function of the logical complexity of the problem - typically characterized as the number of mental-models that need to be evaluated to determine the validity of an inference (see e.g., Johnson-Laird, 2001). For example, logical problems that can be solved by constructing only a single representation of the premises show less belief-bias than more complex problems - that is, arguments which according to mental-model theory require the construction of up to three representations to definitively determine their logical validity ${ }^{1}$ (Klauer, Musch, \& Naumer, 2000; Newstead, Pollard, Evans, \& Allen, 1992; Oakhill, Johnson-Laird, \& Garnham, 1989; Trippas, Handley, \& Verde, 2013). The fact that belief bias tends to be reduced on simpler problems is consistent with the hypothesis that

\footnotetext{
${ }^{1}$ Even though we draw on mental-model theory as a means of defining problem complexity, we do not rule out the possibility that alternative mental representations or reasoning strategies are being used by our participants (e.g., probability heuristics: Chater \& Oaksford, 1999; mental logic: Braine \& O'Brien, 1991). Furthermore, these alternative frameworks also allow for the characterization of inferential complexity in a way which generally maps on to the one provided by mental-model theory.
} 
judgments of validity are completed more quickly and thus that beliefs have less of an opportunity to interfere with a rapidly generated logical response. On the other hand, the evidence that belief bias increases with complexity is not always consistent (Evans \& Pollard, 1990).

The goal of the current paper is to provide a direct test of the complexity hypothesis by varying the difficulty of the logical task, and asking participants to evaluate logical validity and conclusion believability. We predicted that the degree to which logical validity and belief judgments interfere with each other will depend on the complexity of the processes required to render them. If the logical structures are extremely simple, then we expected to replicate past findings and show that validity interferes more with belief-judgments than vice versa (Handley et al., 2011). As logical complexity increases, this asymmetry should be reduced, and in fact, should be reversed for the most complex logical problems, where believability should interfere more with logic judgments than viceversa.

\section{Experiment 1}

In Experiment 1, our aim was to replicate and extend the findings by Handley et al. (2011) to a set of more difficult conditionals (modus tollens: If $\mathrm{p}$, then q; not $\mathrm{q}$, therefore not p). Participants were given a set of problems that included both modus ponens and modus tollens inferences and asked to judge whether the conclusion was logically valid half the time or believable the other (see also Johnson-Laird \& Byrne, 2002, for a mental-models account of how people reason about such conditional inferences). On half of the trials, logical analysis and belief analysis produced the same response (no-conflict trials: i.e., valid-believable and invalid-unbelievable) and on the other half, they produced different responses (conflict trials: i.e., valid-unbelievable and invalid-believable). The modus ponens trials were expected to replicate Handley et al. (2011) in that logic-belief conflicts should have a greater impact on belief judgments than logic judgments. Whereas performance with modus ponens is usually quite high, accuracy is lower for modus tollens (Wason, 1968; see Evans, Newstead, \& Byrne, 1993 for review). Thus, for modus tollens, we predict the asymmetry in complexity to either be reduced or to reverse direction.

\section{Method}

Participants Forty-five undergraduate psychology students from Plymouth University (UK) or the University of Saskatchewan (Canada) participated in exchange for course credit. Thirty-two participants were female and 13 were male (age range $=18-35$ years, $M=19$ ).
Design, materials and measures We used a 2 (belief-logic conflict: conflict vs. no conflict) $\times 2$ (instructions: logic vs belief) $\times 2$ (argument type: modus ponens vs. modus tollens) within subjects design. We created four lists containing 64 arguments each, half of which were modus ponens and half of which were modus tollens, based on 32 distinct item content themes (see Table 1 for examples). We crossed logical validity and conclusion believability to create 16 conflict (valid-unbelievable, invalid-believable) and 16 no-conflict (validbelievable, invalid-unbelievable) items within each argument type. Half of the problems were presented under logic instructions and half were presented under belief instructions. Item contents were counterbalanced by using only half of the themes per item list, half of which were used to create modus ponens problems, and half to create modus tollens problems. Within each argument type each theme was presented four times, once in each conflict by instruction cell. Problem contents were taken and extended from Handley et al. (2011, Exp. 5). We measured accuracy, response time, and confidence on each trial. On each trial, the major (conditional) premise was presented. Upon pressing the spacebar the major premise disappeared and the minor (categorical) premise appeared, as did the conclusion and the response options. The response options acted as the instructional cue: under logic instructions, the response options were "valid" and "invalid"; under belief instructions, the response options were "believable" and "unbelievable." Responses were made by pressing the s-key (valid/believable, depending on instructions) or the k-key (invalid/unbelievable). After each response, we asked the participants to indicate how confident they were that their response was correct on a scale from 1 (guess) to 3 (certain). We also analyzed confidence ratings ${ }^{2}$ as they have been shown to reflect conflict, thus potentially providing converging evidence for the accuracy and response time data (Johnson, Tubau, \& De Neys, 2016). There were 16 practice trials with feedback (not analyzed), and 64 experimental trials (presented in a randomized order for each participant).

Procedure Participants were randomly assigned to one of the four problem randomizations. Before starting the experiment they were briefed about the study, asked to sign a consent form, and presented with the following instructions:

When instructed to answer according to beliefs you must answer according to your knowledge of what is true in the world, for example:

If you finish a drink then the glass will be full.

\footnotetext{
2 The confidence ratings were collected with the aim to conduct signal detection theory (SDT) analyses (cf., Dube, Rotello, \& Heit, 2010; Trippas, Handley, \& Verde, 2014), which we did not report given that they converged with the reported analyses. As a consequence, our confidence rating scale has a very low resolution, potentially negatively impacting the possibility of uncovering meaningful patterns. We report the data for completeness' sake.
} 
Table 1 Experiment 1: Examples of the problems with correct responses

\begin{tabular}{|c|c|}
\hline CONFLICT PROBLEMS & NO-CONFLICT PROBLEMS \\
\hline Valid-Unbelievable & Valid-Believable \\
\hline MP: If a child is happy, then it cries & MP: If a child is happy, then it laughs \\
\hline Suppose a child is happy & Suppose a child is happy \\
\hline Does it follow that the child cries? & Does it follow that the child laughs? \\
\hline MT: If a child is happy, then it cries & MT: If a child is sad, then it cries \\
\hline Suppose a child laughs & Suppose a child laughs \\
\hline Does it follow that the child is sad? & Does if follow that the child is happy? \\
\hline Correct according to logic: YES & Correct according to logic: YES \\
\hline Correct according to beliefs: NO & Correct according to beliefs: YES \\
\hline Invalid - Believable & Invalid - Unbelievable \\
\hline MP: If a child is happy, then it cries & MP: If a child is happy, then it laughs \\
\hline Suppose a child is happy & Suppose a child is happy \\
\hline Does it follow that the child laughs? & Does it follow that the child cries? \\
\hline MT: If a child is happy, then it cries & MT: If a child is sad, then it cries \\
\hline Suppose a child laughs & Suppose a child laughs \\
\hline Does it follow that the child is happy? & Does it follow that the child is sad? \\
\hline Correct according to logic: NO & Correct according to logic: NO \\
\hline Correct according to beliefs: YES & Correct according to beliefs: NO \\
\hline
\end{tabular}

Note. MP: example of modus ponens inference, MT: example of modus tollens inference

Suppose your glass is empty.

Does it follow that your drink will be full?

The correct answer according to beliefs is UNBELIEVABLE because based upon your knowledge of the world you know that if a drink is finished then the glass will be empty, hence the conclusion is unbelievable. However, when instructed to answer according to logic you must assume each statement is true (even if in reality it is not true) and respond with the answer which logically follows from the statements presented, e.g.:

If you finish a drink then the glass will be full.

Suppose you finish your drink.

Does it follow that your drink is empty?

The correct answer according to logic is INVALID, because the first premise states that "if you finish a drink then the glass will be full" and supposing you "finish your drink" you must logically conclude that your drink will be full. This is why the conclusion "does it follow that your drink is empty" is logically invalid.

After completing the experiment participants were thanked and debriefed by the experimenter.

\section{Results}

Analysis approach We analyzed the accuracy data using a generalized linear mixed model approach with a logit link, binomially distributed residuals, and a random effects structure justified by the experimental design and the data (Barr, Levy, Scheepers, \& Tily, 2013; Bates, Kliegl, Vasishth, \& Baayen, submitted). Random intercepts for participants were included, as were random slopes for the main effects of the withinparticipants manipulations. Failures to converge were addressed by either dropping the random effect which explained the least variance, or by assuming the covariances between the random effects were 0 (these approaches led to identical conclusions unless otherwise noted). Odds ratios (ORs) of the fixed effects coefficients of the full model are reported as effect sizes (Hosmer \& Lemeshow, 2004), as there is considerable debate about how to calculate effect size within the generalized linear mixed model framework with correlated random effects (Nakagawa \& Schielzeth, 2013). We used R for all our analyses (R Core Team, 2015). The mixed function from the afex package (Singmann, Bolker, \& Westfall, 2015) was used to test for all main effects and interactions. This function relies on the lme4 package (Bates, Maechler, Bolker, \& Walker, 2015). Response times and confidence ratings were analyzed in an analogous fashion, with the exception that we logarithmically transformed the response times prior to analyzing the data, and that we assumed normally distributed residuals without a link function. For these analyses we report effect size in terms of Cohen's $d$ calculated from the means and standard deviations of the full model.

Prior to the analyses, two participants were removed because they scored substantially below chance on the conflict items ( $<40 \%$ accuracy), suggesting that they may have misinterpreted the task (i.e., responding on the basis of logic 
under belief instructions and vice versa). An additional 100 observations $(<3.5 \%)$ were flagged as outliers based on response time boxplots and removed.

Accuracy The accuracy data are summarized in Table 2. A 2 (Conflict: conflict vs. no-conflict) $\times 2$ (Instructions: logic vs. belief) $\times 2$ (Problem Type: modus ponens vs. modus tollens) within-participants analysis of accuracy indicated the follow pattern of results: Accuracy was lower for conflict $(M=.68)$ than for no-conflict $(M=.92)$ problems, $\chi^{2}(1)=39.7, p<$ $.0001, O R=2.88$. Accuracy was also lower for modus tollens $(M=.78)$ than for modus ponens $(M=.82)$ problems, $\chi^{2}(1)=$ $18.05, p<.0001, O R=1.33$. Conflict and Problem Type interacted, $\chi^{2}(1)=15.54, p<.0001, O R=1.31$, indicating that the conflict - no-conflict difference was larger for modus ponens (diff $=0.27)$ than for modus tollens $($ diff $=.21)$ problems. Instructions and Problem Type also interacted, $\chi^{2}(1)=$ $12.79, p=.0003, O R=1.28$, indicating that for the modus ponens problems, belief-based accuracy $(M=.79)$ was lower than logic-based accuracy $(M=.85)$, whereas no such difference emerged for the modus tollens problems $\left(M_{\text {belief }}=.79, M\right.$ logic $=.77)$. These effects were qualified by a marginal threeway interaction, $\chi^{2}(1)=3.47, p=.06, O R=1.14 \mathrm{We}$ interpreted this interaction by analyzing the data for the modus ponens and the modus tollens problems separately.

For the modus ponens problems, there was a significant main effect of Conflict $(M$ conflict $=.95, M$ no-conflict $=$ .68), $\chi^{2}(1)=44.11, p<.0001, O R=5.11$, as well as a main effect of Instruction $(M$ logic $=.85, M$ belief $=.79), \chi^{2}(1)=$ $7.35, p=.007, O R=2.23$. Crucially, these factors interacted, $\chi^{2}(1)=5.34, p=.02, O R=1.48$, indicating that belief-logic conflict interfered more with belief judgments $($ diff $=.30)$ than with logic judgments (diff $=.24)$, with both significantly different from 0 , all $p<.001$, all $O R>2.51$.

For the modus tollens problems, there was only a main effect of Conflict $(M$ conflict $=.89, M$ no-conflict $=.68$ ), $\chi^{2}(1)=26.58, p<.0001, O R=2.06$. No other effects approached significance, all $p \mathrm{~s}>.30 .^{3}$ Thus, for the easier modus ponens inference, we replicated earlier findings that logical validity interfered more with belief judgments than vice versa (Handley et al., 2011), but this difference disappeared for the more difficult modus tollens inferences. For these more complex arguments, the interference was symmetrical, with validity interfering with belief judgments and vice versa to a similar degree.

Response time The data are summarized in Table 3. A 2 (Conflict: conflict vs. no-conflict) $\times 2$ (Instructions: logic vs.

\footnotetext{
${ }^{3}$ A simulation of 1,000 experiments assuming 45 participants solving 64 trials each indicated we had good power $(81 \%)$ to detect small effects $(d=0.20$ corresponding to a $5.6 \%$ accuracy difference), conditional on our analysis technique.
}

Table 2 Experiment 1: Mean accuracy (in terms of proportion correct) for each cell of the design

\begin{tabular}{llllll}
\hline & \multicolumn{2}{l}{ Modus Ponens } & & \multicolumn{2}{l}{ Modus Tollens } \\
\cline { 2 - 3 } & Belief & Logic & & Belief & \multicolumn{1}{l}{ Logic } \\
\hline Conflict & $.63(.48)$ & $.73(.45)$ & & $.67(.47)$ & $.69(.46)$ \\
No Conflict & $.93(.25)$ & $.97(.17)$ & & $.91(.29)$ & $.86(.35)$ \\
Difference & .30 & .24 & & .24 & .17 \\
\hline
\end{tabular}

Note. Standard deviations between brackets

belief) $\times 2$ (Problem Type: modus ponens vs. modus tollens) within-participants analysis of log-transformed response time indicated the following pattern of results: Conflict significantly slowed down responding $\left(M_{\text {conflict }}=4786 \mathrm{~ms}, M_{\text {no-conflict }}\right.$ $4503 \mathrm{~ms}$, geometric means), $\chi^{2}(1)=7.71, p=.006, d=0.17$. Responding was also slower for modus tollens problems $(M=$ $4853 \mathrm{~ms})$ than for modus ponens problems $(M=4445 \mathrm{~ms})$, $\chi^{2}(1)=48.07, p<.0001, d=0.27$. This latter finding is consistent with our assumption that the processes required to generate modus tollens inferences are more complex than those required to make modus ponens inferences. No other effects approached significance, all $p \mathrm{~s}>.18$. Thus, the asymmetry in the effect of conflict on belief and logic judgments for modus ponens was not observed in the response time data. Although some other studies have observed such an asymmetry in response times, these findings are typically less consistent than those from the accuracy data (Handley et al., 2011; Pennycook et al., 2014). Nevertheless, in keeping with those data and other published work (e.g., De Neys \& Glumicic, 2008; Thompson et al., 2011), response times on the conflict problems were longer than the non-conflict problems.

Confidence ratings The data are summarized in Table 4. A 2 (Conflict: conflict vs. no-conflict) $\times 2$ (Instructions: logic vs. belief) $\times 2$ (Problem Type: modus ponens vs. modus tollens)

Table 3 Experiment 1: Mean response time (in milliseconds) for each cell of the design

\begin{tabular}{llllll}
\hline & \multicolumn{2}{l}{ Modus Ponens } & & \multicolumn{2}{l}{ Modus Tollens } \\
\cline { 2 - 3 } \cline { 5 - 6 } \cline { 5 - 6 } & Belief & Logic & & Belief & \multicolumn{2}{l}{ Logic } \\
\hline Conflict & $4615(1497)$ & $4516(1479)$ & $5117(1829)$ & $4935(1702)$ \\
No Conflict & $4418(1477)$ & $4241(1390)$ & & $4664(1536)$ & $4717(1641)$ \\
Difference & 197 & 275 & 453 & 218 \\
\hline
\end{tabular}

Note. Standard deviations between brackets. Although we analyzed $\operatorname{logRTs}$, here we wanted to present the data in the original units. For this purpose, we report geometric means (i.e., $\exp (\operatorname{mean}(\log (\mathrm{RT})))$ )). Corresponding geometric standard deviations are reported. These were calculated by subtracting one standard deviation of $\log (\mathrm{RT})$ from the mean $\log (\mathrm{RT})$, and taking $\exp ($.$) of the result. The resulting value was$ then subtracted from the geometric mean to get an equivalent geometric standard deviation in units of $\mathrm{ms}$ 
Table 4 Experiment 1: Mean confidence rating for each cell of the design

\begin{tabular}{llllll}
\hline & \multicolumn{2}{l}{ Modus Ponens } & & \multicolumn{2}{l}{ Modus Tollens } \\
\cline { 2 - 3 } \cline { 5 - 6 } \cline { 5 - 6 } & Belief & Logic & & Belief & Logic \\
\hline Conflict & $2.45(0.70)$ & $2.35(0.70)$ & & $2.38(0.74)$ & $2.21(0.74)$ \\
No Conflict & $2.55(0.65)$ & $2.59(0.59)$ & & $2.50(0.65)$ & $2.34(0.74)$ \\
Difference & 0.10 & 0.24 & & 0.12 & 0.13 \\
\hline
\end{tabular}

Note. Standard deviations between brackets. The scale ranged from 1 (least confident) to 3 (most confident)

within-participants analysis of the confidence ratings (on a scale from $1=$ least confident, to 3 = most confident) demonstrated that people were less confident when logic and belief were in conflict compared to when this was not the case $(M$ conflict $\left.=2.35, M_{\text {no conflict }}=2.50\right), \chi^{2}=13.38, \mathrm{df}=1, p=.0003$, $d=0.23$. People were significantly more confident making belief-based than logic-based judgments $\left(M_{\text {belief }}=2.47, M\right.$ logic $=2.37), \chi^{2}=7.01, \mathrm{df}=1, p=.008, d=0.17$, which may be surprising given that, if anything, accuracy was higher under logic instructions. Confidence was also lower for the modus tollens than the modus ponens problems $\left(M_{\mathrm{MP}}=2.48\right.$, $\left.M_{\mathrm{MT}}=2.36\right), \chi^{2}=27.51, \mathrm{df}=1, p<.0001, d=0.21$. Finally, Instructions and Problem type also interacted, $\chi^{2}=7.15, \mathrm{df}=$ $1, p=.008, d=0.21$, suggesting that people were equally confident making belief and logic judgments for modus ponens problems $\left(M_{\text {belief }}=2.50, M_{\text {logic }}=2.47, p=.39, d=\right.$ 0.06 ), but significantly less confident making logic than belief judgments for modus tollens problems $\left(M_{\text {belief }}=2.44, M_{\text {logic }}\right.$ $=2.27, p=.0005, d=0.26$ ). This suggests that belief-based judgments were comparable for modus ponens and modus tollens, but that logic judgments were more affected for modus tollens, supporting the accuracy and response time results showing that making a logic-based judgment is more difficult for modus tollens than modus ponens. No other effects approached significance, all $p \mathrm{~s}>.16$.

\section{Discussion}

As predicted, we found that belief-logic conflict interferes more with belief judgments than with logic judgments for modus ponens, but not when reasoning about the more complex modus tollens. In contrast, for the modus tollens inference, the interference was bidirectional: logical validity interfered with belief-judgments to the same extent that argument believability interfered with logic judgments. Taken together, the findings are consistent with the prediction of the parallelprocessing model that complexity of the relevant problem features determines the nature and degree of interference (Handley \& Trippas, 2015).

The response time and confidence findings provided converging evidence for this interpretation: the slower responding and decreased confidence for modus tollens compared to modus ponens verifies that the former arguments are more complex. The apparent disconnect between the accuracy and confidence findings as a function of problem type is consistent with previous findings in the metacognitive literature suggesting that the correlation between confidence and accuracy is very moderate and affected by several alternative variables, such as a feeling of rightness and processing fluency (Prowse Turner \& Thompson, 2009; Shynkaruk \& Thompson, 2006).

Though not impossible, it seems challenging to reconcile these findings within the default-interventionist framework, which is built upon the assumption that in the deductive reasoning paradigm, beliefs are retrieved in an autonomous fashion - in contrast to logic, the computation of which requires working memory (Evans \& Stanovich, 2013b). On this view, beliefs form a fast, default response that may not be overridden by an attempt to reason logically. As a consequence, one would expect the autonomous, belief-based processing to interfere with the slower, logic-based processing, but not viceversa; this should be particularly true of the modus tollens inference, which is believed to require more complex computations to derive than the modus ponens inference. However, one might argue that the modus tollens inferences, while requiring somewhat longer to process than the modus ponens inference, were still computed quickly enough so that they interfered with belief judgments. Indeed, as the data in Table 3 indicate, latencies for the belief and logic judgments were very similar in the case of the modus tollens inference, suggesting that they required similar levels of processing effort. In the next study we increased the complexity of the logical arguments. According to the parallel-processing model, doing so should reverse the pattern of results reported here.

\section{Experiment 2}

In Experiment 1, we demonstrated that belief-logic conflict interferes more with belief than with logic judgments, but that this effect is eliminated when the complexity of the logical argument is increased - presumably equating it to the complexity of the belief judgment. In the current study, we took the next logical step by further increasing the complexity of the logical structure. The parallel-processing model predicts that increased logical complexity should lead to a reversal of the effect. In other words, belief-logic conflict should interfere more with logic judgments than with belief judgments (Handley \& Trippas, 2015).

We tested our prediction in a syllogistic reasoning task. Participants were presented with simple and complex syllogisms. The complexity of the syllogisms was determined on a theoretical basis as well as on an empirical one. Theoretically, the two leading models of syllogistic reasoning suggest that our simple syllogisms should be easier than the difficult ones, 
either because the simple syllogisms were all one-model syllogisms, whereas the complex syllogisms were multiplemodel ones (Johnson-Laird \& Byrne, 1991), or because the simple syllogisms require fewer and simpler heuristics to solve (Chater \& Oaksford, 1999). This theoretical analysis is backed up by empirical findings that the simpler syllogisms are solved more accurately than the complex ones (Klauer et al., 2000; Trippas et al., 2013).

The simple syllogisms were hypothesized to serve a similar role to the modus tollens conditionals in Experiment 1, suggesting we can expect roughly equal interference for belief and logic judgments. For the complex syllogisms, making correct logical judgments will become more difficult. Thus, we predict the opposite pattern of results observed in Experiment 1: for simple syllogisms, we expected similar levels of belief-logic conflict interference for belief and logic judgments. For complex syllogisms, conflict should interfere more for logic than for belief judgments.

\section{Method}

Participants Eighty-four undergraduate psychology students from the University of Saskatchewan (Canada) participated in exchange for course credit. Fifty-three participants were female and 31 were male (age range $=18-60$ years, $M=22$ ).

Design, materials and measures We used a 2 (Belief-logic Conflict: conflict vs. no conflict) $\times 2$ (Instructions: logic vs. belief) $\times 2$ (Problem Type: simple syllogisms vs. complex syllogisms) within-participants design. Problem contents were randomly paired with logical structures as in Trippas et al. (2013). Examples in each cell of the design can be found in Table 5. We crossed logical validity and conclusion believability to create 16 conflict (valid-unbelievable, invalid-believable) and 16 noconflict (valid-believable, invalid-unbelievable) items within each level of syllogism complexity. Half of the problems were presented under logic instructions and half were presented under belief instructions. Problem contents were taken and developed from Trippas et al. (2013, Exp. 1) (see Table 5 for examples). We measured choice, response time, and confidence. On each trial, the premises were initially presented for a fixed period. After $3 \mathrm{~s}$ the conclusion was also presented, together with the response options, and an instructional cue at the top of the screen stating either BELIEF or LOGIC in red. This approach was taken to ensure that the design did not unfairly favor beliefs by permitting a shortcut strategy where participants could simply evaluate the conclusion believability without considering the premises. The response options acted as an additional instructional cue: under logic instructions, the response options were "valid" and "invalid"; under belief instructions, the response options were "believable" and "unbelievable." Responses were made by pressing the s-key (valid/believable, depending on the instructional set on the current trial) or the k-key (invalid/unbelievable).
After each response, we asked the participants to indicate how confident they were that their response was correct on a scale from 1 (guess) to 3 (certain). There were 16 practice trials with feedback (not analyzed), and 64 experimental trials (presented in a randomized order for each participant).

Procedure The procedure was identical to the one in Experiment 1, with the exception of the instructions, which now read:

In this experiment, we are interested in your ability to make two types of judgments: judgments on the basis of LOGIC, and judgments on the basis of BELIEFS. When the word "LOGIC" appears in red at the top of the screen, you should assume all the information ABOVE the line is true (even if it's not, or if it doesn't appear to make much sense). After a short amount of time, a conclusion sentence BELOW the line will appear, which you will be asked about. If you judge that the conclusion necessarily follows from the premises, you should answer "Valid" by pressing the "s"-key, otherwise you should answer "Invalid" by pressing the "k"key. For example:

All cars are blurbs

All blurbs are cheap

All cars are cheap

Given the instruction to respond on the basis of LOGIC, you should respond "Valid," because the sentence "All cars are cheap" necessarily follows from the premises above the line (if you assume they are true). When the word "BELIEF" appears in red at the top of the screen, you should focus on whether the information is in line with your beliefs about what is true in the world. If you think the information BELOW the line is consistent with your knowledge of the world, you should respond "Believable" by pressing the "s"-key. Otherwise, please respond "Unbelievable" by pressing the "k"-key. For example:

All cars are blurbs

All blurbs are cheap

All cars are cheap

Given the instruction to respond on the basis of BELIEF, you should respond "Unbelievable" because you presumably know from your experience of the world that the sentence "All cars are cheap" is false (consider, for instance, the cost of a Ferrari or a Porsche).

\section{Results}

Analysis approach The analyses were performed in the same manner as in Experiment 1. Two participants were excluded based on their substantial $(<40 \%)$ below-chance accuracy performance on the conflict items, indicating that they were not 
Table 5 Experiment 2: Examples of the problems with correct responses

CONFLICT PROBLEMS

Valid - Unbelievable

Simple: All drinks are dralys

No dralys are beers

No beers are drinks

Complex: No boats are stamuses

Some yachts are stamuses

Some yachts are not boats

Correct according to logic: YES

Correct according to beliefs: NO

Invalid - Believable

Simple: All willows are glukers

No glukers are trees

Some willows are trees

Complex: No amphibians are vindeces

Some frogs are vindeces

Some amphibians are not frogs

Correct according to logic: NO

Correct according to beliefs: YES

NO CONFLICT PROBLEMS

Valid-Believable

Simple: All salmons are vennars

No venners are fruits

No salmons are fruits

Complex: No murderers are catepies

Some criminals are categpies

Some criminals are not murderers

Correct according to logic: YES

Correct according to beliefs: YES

Invalid - Unbelievable

Simple: All dalmatians are curges

No vegetables are curges

Some vegetables are Dalmatians

Complex: No spears are cortemns

Some weapons are cortemns

Some spears are not weapons

Correct according to logic: NO

Correct according to beliefs: $\mathrm{NO}$ engaging with the task. An additional 15 responses $(<.01 \%)$ were classified as outliers based on a boxplot of logtransformed response time and excluded.

Accuracy The data are summarized in Table 6. A 2 (Conflict: conflict vs. no-conflict) $\times 2$ (Instructions: logic vs. belief) $\times 2$ (Problem Type: Simple vs. Complex) within-participants analysis of accuracy indicated the follow pattern of results: as expected, accuracy was lower for conflict $(M=.68)$ than for no-conflict $(M=.83)$ problems, $\chi^{2}(1)=60.45, p<.0001, O R=1.58$, and lower for complex syllogisms $(M=.71)$ than for simple ones $(M$ $=.80), \chi^{2}(1)=66.93, p<.0001, O R=1.35$. As we predicted, and in contrast to Experiment 1, accuracy was lower under logic instructions $(M=.71)$ than under belief instructions $(M=.80)$, $\chi^{2}(1)=21.23, p<.0001, O R=1.32$. The predicted interaction between Conflict and Instruction was significant, $\chi^{2}(1)=4.34, p$ $=.04, O R=1.08$, indicating that belief-logic conflict had a larger effect under logic instructions (diff $=.20$ ) than under belief

Table 6 Experiment 2: Mean accuracy (in terms of proportion correct) for each cell of the design

\begin{tabular}{llllll}
\hline & \multicolumn{2}{l}{ Simple } & & & \multicolumn{2}{l}{ Complex } & \\
\cline { 2 - 3 } \cline { 5 - 6 } & Belief & Logic & & Belief & Logic \\
\hline Conflict & $.73(.45)$ & $.72(.45)$ & & $.76(.43)$ & $.50(.50)$ \\
No Conflict & $.87(.33)$ & $.90(.30)$ & & $.85(.36)$ & $.71(.45)$ \\
Difference & 0.14 & 0.18 & & 0.09 & 0.21 \\
\hline
\end{tabular}

Note. Standard deviations between brackets instructions $($ diff $=.12)$ : the reverse of the pattern observed in Experiment 1. There was an interaction between Conflict and Problem Type, $\chi^{2}(1)=4.54, p=.03, O R=1.08$, indicating that the effect of conflict was larger for simple problems $($ diff $=.17)$ than for complex problems (diff $=.15$ ), though the difference was numerically small. Finally, Instruction and Problem Type interacted, $\chi^{2}(1)=68.57, p<.0001, O R=1.36$, suggesting that for the simple problems, accuracy under belief and logic instructions was similar $\left(M_{\text {belief }}=.80, M_{\text {logic }}=.81\right)$, whereas for the complex problems, accuracy under belief instructions was much higher $(M=.81)$ than under logic instructions $(M=.61)$. Although the three-way interaction was not significant $(p=.42$, $O R=1.03$ ), we decided to analyze the simple and the complex problems separately for three reasons: (1) to aid interpretation of the complex interactive pattern described above, (2) for reasons of a priori theoretical interest, and (3) for congruency with the findings reported in Experiment 1.

For the simple arguments, there was a significant main effect of Conflict $\left(M_{\text {conflict }}=.72, \mathrm{M}_{\text {no-conflict }}=.89\right), \chi^{2}(1)=$ 69.90, $p<.0001, O R=1.91$. No other effects approached significance, $p \mathrm{~s}>.15$. $^{4}$ Thus, like the MT problems in Experiment 1, there was a symmetric effect of conflict for these problems, with validity interfering with beliefs to about the same extent as the reverse.

\footnotetext{
${ }^{4}$ A simulation of 1,000 experiments assuming 85 participants solving 64 trials each indicated we had excellent power $(>98 \%)$ to detect small effect $(\mathrm{d}=0.20$ corresponding to a $5.6 \%$ accuracy difference), conditional on our analysis technique.
} 
For the complex arguments, there was a significant main effect of Conflict $(M$ conflict $=.78, M$ no-conflict $=.63), \chi^{2}(1)$ $=25.72, p<.0001, O R=1.40$. There was also a significant main effect of Instruction $(M \operatorname{logic}=.61, M$ belief $=.81), \chi^{2}(1)$ $=69, p<.0001, O R=1.81$. Crucially, Conflict and Instruction interacted, $\chi^{2}(1)=4.86, p=.03, O R=1.14$, indicating that belief-logic conflict interfered more with logic judgments (diff $=.21)$ than with belief judgments $($ diff $=.09)$, with both different from 0 , all $p \leq .03$, all $O R>1.23$.

This pattern mirrored the one observed in Experiment 1. In that experiment, belief-logic conflict interfered more with belief judgments than logic judgments, but only on the simplest arguments. Here, conflict interfered with logic judgments more than belief judgments, but only on the most complex arguments. Thus, when the logical structures are very simple (modus ponens), conflict interferes with judgments based on belief. When the logical structures are of moderate complexity (modus tollens and simple syllogisms), the interference is bidirectional. When the logical structures are complex, conflict interferes more with logic judgments than belief judgments.

To verify this interpretation we analyzed accuracy using a 2 (Instructions: logic vs. belief) $\times 2$ (Complexity: modus ponens/ simple vs. modus tollens/complex) $\times 2$ (Experiment: one [conditionals] vs. two [syllogisms]) analysis of accuracy for the conflict items only. Consistent with the key prediction of the model, a significant three-way interaction between Instructions, Complexity, and Experiment emerged, $\chi^{2}=6.92$, df $=1, p=$ $.009, O R=1.14$. Follow-up tests comparing the effect of Instructions for each Experiment by Complexity cell confirms the specific direction of the interaction: For the simple conditionals, accuracy was higher under logic than under belief instructions, $\chi^{2}=7.44$, df $=1, p=.006, O R=1.28$. For the complex conditionals and the simple syllogisms, there were no statistically significant differences, all $\chi^{2}<0.30, \mathrm{df}=1$, all $p>$ .58 , all $O R<1.06$. Finally, for the complex syllogisms, accuracy under belief instructions is significantly higher than accuracy under logic instructions, $\chi^{2}=107.8, \mathrm{df}=1, p<.0001, O R=1.90$.

Response time The data are summarized in Table 7. A 2 (Conflict: conflict vs. no-conflict) $\times 2$ (Instructions: logic vs. belief) $\times 2$ (Problem Type: Simple vs. Complex) withinparticipants analysis of response time indicated the following pattern of results: conflict slowed down responding $\left(M_{\text {conflict }}=\right.$ $\left.9584 \mathrm{~ms}, M_{\text {no-conflict }}=9188 \mathrm{~ms}\right), \chi^{2}(1)=5.26, p=.02, d=.06$. People responded more slowly under logic instructions $(M=$ $10718 \mathrm{~ms})$ than under belief instructions $(M=8217 \mathrm{~ms}), \chi^{2}(1)$ $=35.79, p<.0001, d=0.42$. People also responded more slowly to complex syllogisms $(M=9902 \mathrm{~ms})$ than to simple syllogisms $(M=8894 \mathrm{~ms}), \chi^{2}(1)=28.59, p<.0001, d=0.17$. Conflict and Problem Type interacted, $\chi^{2}(1)=3.93, p=.05, d=$ 0.11 , suggesting that conflict had a larger impact for the simple problems (diff $=683 \mathrm{~ms}$ ) than for the complex problems instructions (diff $=72 \mathrm{~ms}$ ). Instruction and Problem Type also interacted, $\chi^{2}(1)=28.29, p<.0001, d=0.30$, indicating that for complex problems, logic-based responding $(M=11842 \mathrm{~ms})$ was a lot slower then belief-based responding ( $M=8281 \mathrm{~ms})$. For the simple problems this difference was much less pronounced $(M \operatorname{logic}=9702 \mathrm{~ms}, M$ belief $=8154)$. This analysis partly reinforces the accuracy analyses: for the complex syllogisms, logical judgments were slowed relative to the beliefbased judgments, whereas for the simple problems, logicbased responding did not suffer to the same degree.

Confidence ratings The data are summarized in Table 8. A 2 (Conflict: conflict vs. no-conflict) $\times 2$ (Instructions: logic vs. belief) $\times 2$ (Problem Type: simple vs. complex) withinparticipants analysis of the confidence ratings (on a scale from 1 = least confidence, to 3 = most confident) demonstrated that people were significantly less confident for conflict $(M=2.48)$ than for no-conflict trials $(M=2.54), \chi^{2}=10.65$, df $=1, p=$ $.001, d=0.10$. Participants were more confident responding under believability $(M=2.56)$ than logic instructions $(M=$ 2.46), $\chi^{2}=10.21, \mathrm{df}=1, p=.001, d=0.16$. Participants were also more confident responding to the simple $(M=2.56)$ than to the complex arguments $(M=2.46), \chi^{2}=34.87, \mathrm{df}=1, p<$ $.0001, d=0.17$. Finally, there was also a significant interaction between Instructions and Problem Type, $\chi^{2}=50.58, \mathrm{df}=1, p$ $<.0001, d=0.32$, suggesting that for the simple problems there was no difference in confidence between logic and belief judgments $(M \operatorname{logic}=2.57, M$ belief $=2.55, p=.47, d=0.04)$.

Table 7 Experiment 2: Mean response time (in milliseconds) for each cell of the design

\begin{tabular}{lllll}
\hline & Simple & & & \multicolumn{2}{l}{ Complex } \\
\cline { 2 - 5 } \cline { 5 - 6 } & Belief & Logic & Belief & Logic \\
\hline Conflict & $8291(4,437)$ & $10305(4,437)$ & $8254(4,532)$ & $11966(6,979)$ \\
No Conflict & $8019(4,275)$ & $9134(4,275)$ & $8309(4,456)$ & $11718(6,788)$ \\
Difference & 272 & 1171 & -55 & 248 \\
\hline
\end{tabular}

Note. Standard deviations between brackets. Although the analysis was based on $\operatorname{logRTs}$, here we wanted to present the data in the original units. For this purpose, we report geometric means (i.e., $\exp (\operatorname{mean}(\log (\mathrm{RT}))))$ ). Corresponding geometric standard deviations are reported. These were calculated by subtracting one standard deviation of $\log (\mathrm{RT})$ from the mean $\log (\mathrm{RT})$, and taking $\exp ($.$) of the result. The resulting value was then subtracted from the$ geometric mean to get an equivalent geometric standard deviation in units of $\mathrm{ms}$ 
Table 8 Experiment 2: Mean confidence rating for each cell of the design

\begin{tabular}{llllll}
\hline & Simple & & & Complex & \\
\cline { 2 - 3 } \cline { 5 - 6 } \cline { 5 - 6 } & Belief & Logic & & Belief & Logic \\
\hline Conflict & $2.52(0.67)$ & $2.54(0.63)$ & & $2.55(0.64)$ & $2.32(0.70)$ \\
No Conflict & $2.57(0.61)$ & $2.60(0.59)$ & & $2.58(0.62)$ & $2.40(0.69)$ \\
Difference & 0.05 & 0.06 & & 0.03 & 0.08 \\
\hline
\end{tabular}

Note. Standard deviations between brackets. The scale ranged from 1 (guess) to 3 (very confident)

By contrast, for the complex syllogisms, people were significantly less confident responding on the basis of logic than on the basis of beliefs $(M$ logic $=2.36, M$ belief $=2.56, p<.0001$, $d=0.36$ ). No other effects approached significance, all $p \mathrm{~s}>$ .24 .

\section{Discussion}

We increased the complexity of the logical judgments and reversed the qualitative pattern of results obtained in Experiment 1. For the simple syllogisms, the effect of conflict was roughly comparable for belief and logic instructions. In contrast, for the complex problems, beliefs interfered with logic judgments more than the reverse. The confidence and response time analyses confirmed that the complex arguments were more difficult and complex to process than the simple ones. We now turn to the general discussion for a more thorough evaluation of the theoretical implications of these findings.

\section{General discussion}

The traditional explanation for many so-called reasoning biases is an assumed asymmetry in the speed and effort with which Type 1 and Type 2 processes are executed. Although processing speed is not considered a defining feature of dual process theories (e.g., Evans \& Stanovich, 2013a), it is typically assumed that belief bias occurs because a quick beliefbased response beats a slower logical analysis (Evans \& Curtis-Holmes, 2005). In contrast to this defaultinterventionist account, the data from the current experiments support the parallel-processing model (Handley \& Trippas, 2015) and other models (De Neys, 2012; Pennycook et al., 2015; Sloman, 2014). These models assume that logic- and belief-based responding is initiated in parallel, rather than in sequence. We also found support for the parallel-processing model's assumption that logical responses can be completed relatively quickly or more slowly depending on their relative complexity. Importantly, this relative complexity was shown to produce predictable patterns of interference.
Specifically, when the logical inference is extremely simple, such as our modus ponens inference in Experiment 1, logical validity interfered with belief-judgments more than believability interfered with logic-judgments (as per Handley et al., 2011). This pattern would not be possible under the assumption that making any type of logical inference takes longer than making belief-based judgments; instead, we interpret this to mean that the processes responsible for drawing the modus ponens inferences finished before those computing the belief judgments, thus interfering with them. Indeed, the data confirmed that logic judgments were made more quickly than belief judgments in that study.

In contrast, for inferences of moderate complexity, such as the modus tollens inferences in Experiment 1 and the simple syllogisms in Experiment 2, the interference was symmetrical. That is, instructions to judge validity interfered with the ability to make judgments based on belief to the same extent that belief instructions interfered with judging validity. Finally, for the most difficult syllogisms in Experiment 2, the interference was once again asymmetrical, but this time, conclusion believability interfered more with validity judgments than vice-versa; indeed, performance for conflict items under logic instructions was at chance levels.

These data support a parallel-processing model, whereby multiple sets of processes are initiated in tandem (Handley \& Trippas, 2015; Newman, Gibb, \& Thompson, 2017; Pennycook et al., 2015; Sloman, 2014). In cases where the processes converge on the same response (i.e., the no-conflict trials), accuracy is high and response times are low. In cases where the processes diverge (i.e., the conflict trials), there is the potential for the processes to interfere with each other: response times are higher and accuracy is lower. Interestingly, even for the most difficult syllogisms, we observed that conclusion validity interfered with the ability to make belief judgments. This suggests that enough information about the logical structure of the problem was extracted in time to interfere with the believability judgment of the statement when logic and belief conflicted. The difference was relatively small, however, leaving open the possibility that this might be an effect produced by a relatively small group of very able reasoners. Alternatively, it is possible that there is some other, structural information that is correlated with validity (Chater \& Oaksford, 1999; Klauer and Singmann, 2013) that is interfering with belief-based processing.

Could the current findings be explained by the fact that we relied exclusively on within-participants manipulations? In both experiments participants could not predict whether they would be asked to respond on the basis of logic or beliefs before the response options appeared. It is possible that they dealt with this by computing both the believability and logical status of the argument during stimulus presentation, reporting only the required judgment when the response options appeared. If this is the case, then an alternative explanation for 
our findings is response competition. ${ }^{5}$ Similarly, it may be that the task switching necessitated by our within-subjects manipulation caused the interference. ${ }^{6}$ Although these effects might be present and could explain the conflict effects that we observed, the question remains why asymmetrical interference occurs as a function of problem complexity. If response competition or task switching were the sole drivers of interference in our paradigm, we should find identical effects of conflict regardless of problem complexity and instruction.

Moreover, we have empirical and theoretical reasons to believe they are not the sole explanation for our findings. Handley et al. (2011) and Howarth, Handley, and Walsh (2016) demonstrated that the same interference occurred in a full between-participants comparison. Participants solved the task in a counterbalanced blocked manner, such that in one block only belief-based responses were required, and in the next block only logic-based responses - and vice versa. Comparisons of the first block between participants who judged logic first or belief first showed that the critical interaction was still present. Thus, the fact that the asymmetrical conflict effects are observed in between-participants designs strongly suggests that our findings are not an artefact of our within-participants manipulation.

\section{The persistence-of-belief bias}

If information about logical validity is available from an early stage, how then do we explain belief bias? That is, why do some reasoners apparently ignore readily available logical information in favor of a belief-based response in the face of explicit instructions to reason logically? At this point, we do not have a simple answer to this question, but offer the following alternatives:

1. Belief bias, as a phenomenon, may be the result of averaging over different strategies. For example, Pennycook and Thompson (2012) noted that base-rate neglect (i.e., the tendency to base judgments on descriptive, situation-specific information rather than the base-rate probability of an event) reflects a mixture of two different strategies, which consist of relying on either the base rate or the stereotype. Because the situation-specific strategy is the more common, the mean result is base-rate neglect. Similarly, in the case of logical reasoning, the phenomenon known as belief bias may reflect a mixture of strategies, one that generates answers based on validity and the other which generates answers based on belief; if the latter is more common, then the average result looks like belief bias (see Stupple, Ball, Evans, \& Kamal-Smith, 2011, for a related suggestion based on a response time analysis). The tendency to use one or the other strategy may

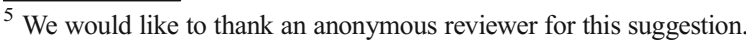

${ }^{6}$ We would like to thank Wim De Neys for this suggestion.
}

depend, amongst other things, on cognitive capacity (Evans, Handley, Neilens, \& Over, 2010; Trippas et al., 2013) or analytic thinking dispositions (Stanovich \& West, 1997; Trippas, Pennycook, Verde, \& Handley, 2015).

2. Answers based on beliefs and logic may differ in their potency or salience. De Neys and colleagues have demonstrated that, in a variety of tasks, there is evidence that reasoners intuitively detect the conflict between formal norms such as logic and probability and beliefs, but often fail to resolve that conflict in favor of the formal norm (see De Neys, 2014 for a summary). His explanation is that beliefs are difficult to inhibit, meaning that belief bias and other phenomena reflect a failure to inhibit a potent, belief-based response in favor of a normative one. A related explanation is essentially Bayesian, namely that reasoners are (rightly) reluctant to set aside beliefs that are based on years of learning in favor of an experimenter's artificial arguments (Evans \& Over, 1996). Indeed, some people would argue that there is no point in striving to attain logical coherence at all costs, but that correspondence (i.e., accuracy in terms of what is true in the world) ought to be the only relevant evaluation metric of inferential performance (see e.g., Hammond, 1996, for an overview of the debate). One potential reason for this is that there is apparently little evidence that giving preference to correspondence (e.g., knowledge of what is true in the real world) over coherence (e.g., adherence to the formal laws of logic) results in substantial costs in the real world (Arkes, Gigerenzer, \& Hertwig, 2015). Regardless of one's perspective on such philosophical matters, the fact remains that apparently people are influenced by both logic and beliefs, and that the latter seems to trump the former more often than not.

3. Answers based on logic may be held with degrees of confidence that may vary both from individual to individual and from inference to inference. That is, some reasoners are more confident overall in their ability to reason, and this predicts the probability that they will provide answers based on logical validity (Markovits, Thompson, \& Brisson, 2015). Confidence in an inference may also vary as a function of the complexity of the calculations required to produce an inference, with more complex calculations engendering a lower degree of confidence. Thus, even though inferences based on validity may be produced, they may be held with low confidence and thus subject to re-evaluation (Thompson, Prowse Turner, \& Pennycook, 2011).

4. Much like false memories in recognition memory (Verde \& Rotello, 2003), belief bias may just be a criterion-shiftdriven response bias as interpreted within the framework of signal detection theory (Dube, Rotello, \& Heit, 2010, 2011; Green \& Swets, 1966; Heit \& Rotello, 2014; though see also Klauer \& Kellen, 2011; Trippas, Verde, Handley, Roser, McNair, \& Evans 2014; Singmann \& Kellen, 2014; Trippas, Verde, \& Handley, 2015, for an extensive debate on the interpretation of belief-driven criterion shifts). According to this 
perspective, participants do not ignore argument strength, but they are simply more likely to accept believable conclusions than unbelievable conclusions - all else being equal. This interpretation is not in conflict with our results given that the signal detection theory model of belief bias is not specified at the processing level. For instance, the model is agnostic as to whether the response criterion is set before or after argument strength is calculated. Furthermore, a question arises with regard to how the model would capture responses under belief instructions. Do we assume that these decisions are based on two distributions of belief-strength, with a logic-based response criterion which shifts according to validity (i.e., a socalled logic-bias, Howarth et al., 2016)? For now, we argue it is safest to rely on the signal detection theory as an excellent measurement model until it is specified in a more dynamic way such that it can also make predictions about the timecourse of processing (e.g., Pleskac \& Busemeyer, 2010).

5. Finally, although our data are challenging to capture within a traditional, default interventionist explanation for belief-bias, they do not rule out this framework definitively (Evans \& Stanovich, 2013a, 2013b), especially when considering the more complex forms of arguments. In Experiment 2, responses based on belief were made substantially faster than responses based on logic, which is consistent with the hypothesis that beliefs form a relatively fast, default response that may not be overturned by a slower, logical analysis (Evans \& Curtis-Holmes, 2005). We also found that belief-logic conflict interfered more with judgments of validity than of belief, which is also consistent with the defaultinterventionist view. Indeed, one explanation that fits the data is that some logical arguments might rely solely on Type 1 processing, whereas others require Type 2 processes; conversely, some types of belief judgments may require Type 2 processing. According to this interpretation, our experiments differed with respect to the degree to which Type 1 and Type 2 processes were necessary to judge logical validity.

We also note that our interpretation rests heavily on the relative speed of belief-based and logic-based processes, which is not the defining feature of Type 1 and Type 2 processes (Evans \& Stanovich, 2013a). However, our data do suggest that some logical processes are autonomous, given that they are initiated even when they contradict the current goal state (i.e., to judge believability), which adds complexity to the classification of Type 1 and Type 2 processes and challenges extant explanations of belief-bias. Moreover, the data are challenging to the default-interventionist account of many reasoning phenomena, which rely on relative speed as the basis of the explanation: faster, Type 1 processes produce a default that is not overturned by slower, Type 2 processes (e.g., Evans, 2007; Kahneman, 2011; Toplak, West, \& Stanovich, 2011).
One argument that has been made against the parallelprocessing structure is that it is wasteful of resources (e.g., Evans, 2007). Why initiate costly Type 2 processes if they are routinely terminated because the faster, Type 1 processes have produced a response? In reply, we need to point out that we are not arguing that the types of conflict that we are observing here necessarily arise from a conflict between Type 1 and Type 2 processes. Instead, we argue that people begin to process both the structural features of the problem and to evaluate the believability of the problem at the same time, drawing simultaneously on Type 1 and 2 processing. In some cases, where the structure is simple, a response based on logic or probability may be generated quickly, by Type 1 processes with only minimal Type 2 involvement. In other cases, such as with our complex syllogisms, it may, indeed, require substantial working memory resources to generate and evaluate a conclusion.

Moreover, whereas there might be a cost associated with the needless engagement of Type 2 processes, there are clear benefits to the simultaneous engagement of multiple Type 1 processes. Redundancy gain refers to the enhanced performance that arises when responses are based on multiple stimuli that converge on a single response, as opposed to a response based on a single stimulus. Although most of the evidence for this phenomenon is derived from relatively simple tasks, there is recent evidence that this phenomenon also applies to complex tasks, such as semantic categorization (Shepherdson \& Miller, 2014). Redundancy gain would allow more efficient processing of the non-conflict trials, wherein responses based on multiple stimuli (beliefs and logic) converged on a single response.

\section{Conclusion}

We observed that the logical validity of a conclusion interfered with reasoners' ability to judge the conclusion's believability, even on complex syllogistic problems. Less surprisingly, we also observed that the believability of a conclusion interfered with judgments of validity. In both cases, the degree of interference varied with the complexity of the logical argument. For simple arguments, logic produced more interference than beliefs. For complex arguments, the reverse was true, and for arguments of moderate complexity, the interference was approximately symmetrical. These data are incompatible with explanations of belief-bias that originate with the assumptions that beliefs form a fast, default response that may not be overturned by logical processing. Instead, they support models in which the processing of validity and believability begins in parallel, and the degree of interference that is observed depends on the relative complexity of the processes needed to deliver answers based on beliefs or logic. 
Author Note The first two authors contributed equally to the production and writing of this paper. The research was supported in part by a Discovery Grant from the Natural Sciences and Engineering Research Council of Canada to Valerie Thompson.

Acknowledgments Open access funding provided by Max Planck Society.

Open Access This article is distributed under the terms of the Creative Commons Attribution 4.0 International License (http:// creativecommons.org/licenses/by/4.0/), which permits unrestricted use, distribution, and reproduction in any medium, provided you give appropriate credit to the original author(s) and the source, provide a link to the Creative Commons license, and indicate if changes were made.

\section{References}

Arkes, H., Gigerenzer, G., \& Hertwig, R. (2015). How bad is incoherence? Decision, 3, 20-39.

Bago, B., \& De Neys, W. (2017). Fast logic? Examining the time course of dual process theory. Cognition, 158, 90-109.

Barr, D. J., Levy, R., Scheepers, C., \& Tily, H. J. (2013). Random effects structure for confirmatory hypothesis testing: Keep it maximal. Journal of Memory and Language, 68, 255-278.

Bates, D., Kliegl, R., Vasishth, S., \& Baayen, H. (submitted). Parsimonious mixed models. Submitted to Journal of Memory and Language. Retrieved from https://arxiv.org/abs/1506.04967

Bates, D., Maechler, M., Bolker, B., \& Walker, S. (2015). Fitting linear mixed-effects models using lme4. Journal of Statistical Software, 67, 1-48. doi:10.18637/jss.v067.i01

Braine, M.D.S. \& O'Brien, D.P. (1991). A theory of if: A lexical entry, reasoning program, and pragmatic principles. Psychological Review, 98, 182-203.

Chater, N., \& Oaksford, M. (1999). The probability heuristics model of syllogistic reasoning. Cognitive Psychology, 38, 191-258.

De Neys, W. (2012). Bias and conflict: A case for logical intuitions. Perspectives on Psychological Science, 7, 28-38.

De Neys, W. (2014). Conflict detection, dual processes, and logical intuitions: Some clarifications. Thinking \& Reasoning, 20, 169-187.

De Neys, W., \& Glumicic, T. (2008). Conflict monitoring in dual process theories of thinking. Cognition, 106, 1248-1299.

Dube, C., Rotello, C. M., \& Heit, E. (2010). Assessing the belief bias effect with ROCs: It's a response bias effect. Psychological Review, $117,831-863$.

Dube, C., Rotello, C. M., \& Heit, E. (2011). The belief bias effect is aptly named: A reply to Klauer and Kellen (2011). Psychological Review, 118, 155-163.

Elqayam, S., \& Evans, J. S. B. T. (2011). Subtracting "ought" from "is": Descriptivism versus normativism in the study of human thinking. Behavioral and Brain Sciences, 34, 233-248.

Evans, J. S. B. T. (2007). On the resolution of conflict in dual process theories of reasoning. Thinking \& Reasoning, 13, 321-339.

Evans, J. S. B. T., Barston, J. L., \& Pollard, P. (1983). On the conflict between logic and belief in syllogistic reasoning. Memory \& Cognition, 11, 295-306.

Evans, J. S. B. T., \& Curtis-Holmes, J. (2005). Rapid responding increases belief bias: Evidence for the dual-process theory of reasoning. Thinking \& Reasoning, 11, 382-389.

Evans, J. S. B. T., Handley, S. J., Neilens, H., \& Over, D. (2010). The influence of cognitive ability and instructional set on causal conditional inference. Quarterly Journal of Experimental Psychology, 63, 892-909.

Evans, J. S. B. T., Newstead, S. E., \& Byrne, R. M. J. (1993). Human reasoning: The psychology of deduction. Hillsdale, NJ: Erlbaum.

Evans, J. S. B. T., \& Over, D. E. (1996). Rationality and reasoning. Hove, England: Psychology Press.

Evans, J. S. B. T., \& Pollard, P. (1990). Belief bias and problem complexity in deductive reasoning. Advances in Psychology, 68, 131-154.

Evans, J. S. B. T., \& Stanovich, K. E. (2013a). Dual-process theories of higher cognition advancing the debate. Perspectives on Psychological Science, 8, 223-241.

Evans, J. S. B. T., \& Stanovich, K. E. (2013b). Theory and metatheory in the study of dual processing: Reply to comments. Perspectives on Psychological Science, 8, 263-271.

Green, D., \& Swets, J. (1966). Signal detection theory and psychophysics. New York: John Wiley \& Sons.

Hammond, K. (1996). Upon reflection. Thinking \& Reasoning, 2, 239-248.

Handley, S. J., Newstead, S. E., \& Trippas, D. (2011). Logic, beliefs, and instruction: A test of the default interventionist account of belief bias. Journal of Experimental Psychology: Learning, Memory, and Cognition, 37, 28-43.

Handley, S. J., \& Trippas, D. (2015). Dual processes and the interplay between knowledge and structure: A new parallel processing model. Psychology of Learning and Motivation, 62, 33-58.

Heit, E., \& Rotello, C. M. (2014). Traditional difference-score analyses of reasoning are flawed. Cognition, 131, 75-91.

Hosmer, D. W., \& Lemeshow, S. (2004). Applied logistic regression. New York: John Wiley \& Sons.

Howarth, S., Handley, S. J., \& Walsh, C. (2016). The logic-bias effect: The role of effortful processing in the resolution of belief-logic conflict. Memory \& Cognition, 44, 330-349.

Johnson, E. D., Tubau, E., \& De Neys, W. (2016). The doubting System 1: Evidence for automatic substitution sensitivity. Acta Psychologica, 164, 56-64.

Johnson-Laird, P. N. (2001). Mental models and deduction. Trends in Cognitive Sciences, 5, 434-442.

Johnson-Laird, P. N., \& Byrne, R. M. J. (1991). Deduction. Hillsdale, NJ: Erlbaum.

Johnson-Laird, P. N., \& Byrne, R. M. J. (2002). Conditionals: A theory of meaning, pragmatics, and inference. Psychological Review, 109, 646-678.

Kahneman, D. (2011). Thinking, Fast and Slow. New York: Farrar, Strauss, Giroux.

Klauer, K. C., \& Kellen, D. (2011). Assessing the belief bias effect with ROCs: Reply to Dube, Rotello, and Heit (2010). Psychological Review, 118, 164-173.

Klauer, K. C., Musch, J., \& Naumer, B. (2000). On belief bias in syllogistic reasoning. Psychological Review, 107, 852-884.

Klauer, K. C., \& Singmann, H. (2013). Does logic feel good? Testing for intuitive detection of logicality in syllogistic reasoning. Journal of Experimental Psychology: Learning, Memory, and Cognition, 39, $1265-1273$.

Kruglanski, A., \& Gigerenzer, G. (2011). Intuitive and deliberate judgments are based on common principles. Psychological Review, 118, 97-109.

Markovits, H., Thompson, V. A., \& Brisson, J. (2015). Metacognition and abstract reasoning. Memory \& Cognition, 43, 681-693.

Morsanyi, K., \& Handley, S. J. (2012). Logic feels so good-I like it! Evidence for intuitive detection of logicality in syllogistic reasoning. Journal of Experimental Psychology: Learning, Memory, and Cognition, 38, 596-616.

Nakagawa, S., \& Schielzeth, H. (2013). A general and simple method for obtaining $\mathrm{R}^{\wedge} 2$ from generalized linear mixed-effects models. Methods in Ecology and Evolution, 4, 133-142.

Newman, I.R., Gibb, M., \& Thompson, V.A. (2017). Rule -based reasoning is fast and belief-based reasoning can be slow: Challenging current explanations of belief-bias and base-rate 
neglect. Journal of Experimental Psychology: Learning, Memory, \& Cognition, in press.

Newstead, S. E., Pollard, P., Evans, J. S. B., \& Allen, J. L. (1992). The source of belief bias effects in syllogistic reasoning. Cognition, 45, 257-284.

Oakhill, J., Johnson-Laird, P. N., \& Garnham, A. (1989). Believability and syllogistic reasoning. Cognition, 31, 117-140.

Pennycook, G., Fugelsang, J. A., \& Koehler, D. J. (2015). What makes us think? A three-stage dual-process model of analytic engagement. Cognitive Psychology, 80, 34-72.

Pennycook, G., \& Thompson, V. A. (2012). Reasoning with base rates is routine, relatively effortless, and context dependent. Psychonomic Bulletin \& Review, 19, 528-534.

Pennycook, G., Trippas, D., Handley, S. J., \& Thompson, V. A. (2014). Base rates: Both neglected and intuitive. Journal of Experimental Psychology: Learning, Memory, and Cognition, 40, 544-554.

Pleskac, T., \& Busemeyer, J. R. (2010). Two-stage dynamic signal detection: A theory of choice, decision time, and confidence. Psychological Review, 117, 864-901.

Prowse Turner, J. A., \& Thompson, V. A. (2009). The role of training, alternative models, and logical necessity in determining confidence in syllogistic reasoning. Thinking \& Reasoning, 15, 69-100.

R Core Team (2015). R: A language and environment for statistical computing. R Foundation for Statistical Computing, Vienna, Austria. https://www.R-project.org/

Reder, L. M. (1982). Plausibility judgments versus fact retrieval: Alternative strategies for sentence verification. Psychological Review, 89, 000-000.

Shepherdson, P., \& Miller, J. (2014). Redundancy gain in semantic categorisation. Acta Psychologica, 148, 96-106.

Shynkaruk, J. M., \& Thompson, V. A. (2006). Confidence and accuracy in deductive reasoning. Memory \& Cognition, 34, 619-632.

Singmann, H., Bolker, B., \& Westfall, J. (2015). afex: Analysis of Factorial Experiments. $R$ package version 0.15-2. https://CRAN.Rproject.org/package $=$ afex

Singmann, H. \& Kellen, D. (2014). Concerns with the SDT approach to causal conditional reasoning: A comment on Trippas, Handley, Verde, Roser, McNair, and Evans (2014). Frontiers in Psychology, 5. doi:10.3389/fpsyg.2014.00402

Sloman, S. A. (2014). Two systems of reasoning, an update. In Sherman, J., Gawronski, B., \& Trope, Y. (Eds.). Dual process theories of the social mind. New York: Guilford Press.

Stanovich, K. E., \& West, R. F. (1997). Reasoning independently of prior belief and individual differences in actively open-minded thinking. Journal of Educational Psychology, 89, 342.

Stupple, E. J. N., Ball, L. B., Evans J. St. B. T., \& Kamal-Smith, E. (2011). When logic and belief collide: Individual differences in reasoning times support a selective processing model. Journal of Cognitive Psychology, 23, 931-941.

Thompson, V., \& Evans, J. S. B. T. (2012). Belief bias in informal reasoning. Thinking \& Reasoning, 18, 278-310.

Thompson, V. A., Prowse Turner, J. A. P., \& Pennycook, G. (2011). Intuition, reason, and metacognition. Cognitive Psychology, 63, $107-140$.

Toplak, M. E., West, R. F., \& Stanovich, K. E. (2011). The Cognitive Reflection Test as a predictor of performance on heuristics-andbiases tasks. Memory \& Cognition, 39, 1275-1289.

Trippas, D., Handley, S. J., Verde, M. F., \& Morsanyi, K. (2016). Logic Brightens My Day: Evidence for Implicit Sensitivity to Logical Validity. Journal of Experimental Psychology: Learning, Memory, and Cognition. doi:10.1037/xlm0000248

Trippas, D., Handley, S. J., \& Verde, M. F. (2013). The SDT model of belief bias: Complexity, time, and cognitive ability mediate the effects of believability. Journal of Experimental Psychology: Learning, Memory, and Cognition, 39, 1393-1402.

Trippas, D., Handley, S. J., \& Verde, M. F. (2014). Fluency and belief bias in deductive reasoning: New indices for old effects. Frontiers in Psychology, 5, 631.

Trippas, D., Pennycook, G., Verde, M. F., \& Handley, S. J. (2015). Better but still biased: Analytic cognitive style and belief bias. Thinking \& Reasoning, 21, 431-445.

Trippas, D., Verde, M. F., Handley, S. J., Roser, M. E., McNair, N. A., \& Evans, J. St. B. T. (2014). Modeling causal conditional reasoning data using SDT: caveats and new insights. Frontiers in Psychology, 5. doi:10.3389/fpsyg.2014.00217

Trippas, D., Verde, M. F., \& Handley, S. J. (2015). Alleviating the concerns with the SDT approach to reasoning: reply to Singmann and Kellen (2014). Frontiers in Psychology, 6. doi:10.3389 /fpsyg.2015.00184

Tversky, A., \& Kahneman, D. (1973). Availability: A heuristic for judging frequency and probability. Cognitive Psychology, 5, 207-232.

Verde, M. F., \& Rotello, C. M. (2003). Does familiarity change in the revelation effect? Journal of Experimental Psychology: Learning, Memory, and Cognition, 29, 739-746.

Wason, P. C. (1968). Reasoning about a rule. The Quarterly Journal of Experimental Psychology, 20, 273-281.

Wilkins, M. C. (1929). The effect of changed material on ability to do formal syllogistic reasoning. Archives of Psychology, 102, 83.

Wiswede, D., Koranyi, D., Mueller, F., Langner, O., \& Rothermund, K. (2013). Validating the truth of propositions: Behavioural and ERP indicators of truth evaluation processes. Social Cognitive and Affective Neuroscience, 8, 647-653. 